\title{
A Framework for Context-Sensitive Coordination of Human Interruptions in Human-Computer Interaction
}

\author{
Sonja Gievska ${ }^{1}$, John Sibert ${ }^{1}$ \\ ${ }^{1}$ Department of Computer Science, The George Washington University, \\ Washington D.C. 200052, USA \\ \{sonjag, sibert\}@gwu.edu
}

\begin{abstract}
Recent trends in software development directed toward intelligence, distribution, and mobility need to be followed by an increased sophistication in user interface design. Employment of theoretically sound methods for managing and coordinating complex information, and supporting graceful switching between tasks is especially critical for information-intensive and safety-critical tasks. This paper presents a framework for computer-mediated coordination of human interruptions. As a basis for the framework a new Interruption Taxonomy is outlined to categorize a variety of traceable information needed to exhaustively describe the context space. An exploratory user study is underway to calibrate the kind of benefit gained with the formulated Interruption Model. The expressiveness of the proposed Interruption Model is demonstrated by concretizing the general approach using the particularities of the selected problem domain. The initial results have shown that taxonomy-based coordination of interruption resulted in statistically significant improvement of the primary task resumption time.
\end{abstract}

\section{Introduction}

Any information presented to a user while she is working on a task could be considered interruption of her current endeavor. Given the frequency of presenting information in today's computer systems and the relative scarcity of humans, it is not surprising that some of these interruptions will have disruptive consequences on the human activity being interrupted. Interruption of one task in order to carry out another task very often leads to an increased error rate, hesitation and delay in making decisions, and in general, people are less effective when exposed to interruptions [1], [2], [3], and [7]. When repeated and numerous interruptions arise, increased attentional demand occurs because of the effort required to evaluate the significance of, and decide on appropriate responses to, multiple concurrent events [5]. Persistent and repeated interruptions may affect a person's attitude toward the entire situation, very often leading to a non-optimal behavior. 
Computer outputs are very often generated in isolation from the context in which the interaction occurs. Presenting any kind of information to the user occurs for a reason, and that reason in relation to the current context in a broader sense of view, should ground the relevance and timing of the presentation. Employment of "intelligent" software that knows more about individuals with whom it is interacting and the context of the situation in which the interaction occurs, can help computer systems augment humans' capabilities in the context of interruptions.

The motivation behind this research is the belief that effective coordination of interruption in human-computer interaction (HCI) cannot be accomplished without an appropriate interruption model. The proposed conceptual model is based on the new Interruption Taxonomy and uses Bayesian Belief Networks as a decision-support aid for mediating interruptions. Our first exploratory study is underway and the initial results confirm the hypothesized performance advantage of taxonomy-based coordination of interruption compared to the case with no coordination.

\section{Interruption Taxonomy}

It seems reasonable to establish a taxonomy of important characteristics relevant to the process of coordinating human interruption. The Interruption Taxonomy includes a set of abstractions that helps unify the issues previously considered by other researchers in a variety of different disciplines, proposes new ones, and suggests avenues for further exploration. The interruption-related information (i.e., categories) is categorized according to context: Task Context, User Context, and Environment Context. Each of the dimensions is consistent with a well-established tradition in HCI, which captures the three essential aspects of HCI. A graphical representation of the taxonomy three-dimensional interruption space is presented in Fig.1.

Task-related context knowledge is crucial in disambiguating the meaning and the relevance of the interruption task in regard to the current user endeavor. Interruption requires a coordination of human behavior, and since coordination is "a process of managing dependencies among activities" [6], an important aspect of interruption coordination involves an identification of the relevant task interdependencies.

The user-related categories of the taxonomy are included to support representation of and reasoning about a particular situation as the user views it. Finding solutions to the problems associated with interruptions that are effective with respect to some objective criteria (i.e., task characteristics) is necessary but not sufficient. Coordination solutions must also be acceptable to a user's subjective criteria which may constrain the space of solutions and possible ways of handling interruption.

The Environment Context dimension extends the system context knowledge with information that captures the physicality and the dynamics of the working space. The inclusion of the environment-related categories attributes substantially greater sensitivity to the system, namely, the ability to adapt to a social setting, physical and organizational constraints, or the particularity of a given situation. 


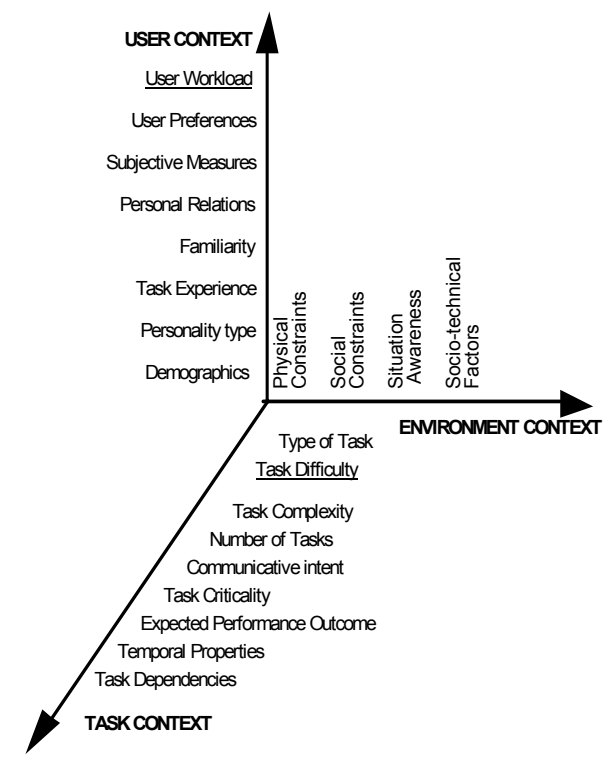

Fig. 1. Interruption Taxonomy

There are two theoretical constructs in the Taxonomy, namely Task Difficulty and User Workload. This framework proposes mapping these categories to other context variables that belong to all three taxonomy dimensions, and include: (1) factors that are used to portray the "objective" difficulty/workload based on what is known about that task in general, (2) characteristics to account for the individual differences and user's perspectives on how difficult/demanding a task is, and (3) environmental influences.

It is our hope that by beginning with the identification and categorization of interruption-relevant context information, this research has in some small way explicated the complex and multidimensional aspects of human interruptions and has attempted to present a way to capture it.

\section{Conceptual Framework}

The conceptual framework for a computer-supported coordination of human interruptions is based on the Interruption Taxonomy. This framework is not an attempt to build a deep psychological model of human behavior under interruptions, but rather an attempt to augment the machine's "intelligence" with a variety of contextual information, and appropriate reasoning machinery that will help the system decide when and in what manner to interrupt users when presenting tasks, messages, alerts and other information. This framework approaches the interruption problem by ana- 
lyzing how people use the circumstances to evaluate the significance of an interruption and plan the most appropriate time to switch between tasks.

Augmenting machine's "intelligence" with a variety of contextual information, and appropriate reasoning machinery can help a system decide when and in what manner to interrupt users when presenting information. Inferential techniques must be applied to provide context-sensitive actions that are responsive to a particular state of interaction. We have adopted an approach based on Bayesian Belief Networks (BBNs) to represent the causal relationship between different pieces of information and to integrate rules for how to use, maintain, and reason with interruption-related knowledge. BBNs have proved suitable for making predictions and decisions from incomplete and uncertain data [4]. For our first exploratory study, the constructed BBN shown in Fig. 2 was initialized and trained off-line. Its outcomes were used as a basis for implementing the taxonomy-based mediated control of interruptions in our experimental environment.

\section{Exploratory User Study}

Our major priority at the moment is to gather some evidence to evaluate the adequacy of the proposed approach and the utility of the taxonomy. To demonstrate the scope and the utility of the Interruption Taxonomy, an exploratory user study is underway. Our goal is to demonstrate the expressiveness of the Taxonomy by applying it in a particular interruption environment. In addition, we examined the effectiveness of taxonomy-based mediated control of interruptions compared to condition with no interruption control (i.e., interruptions were presented immediately at randomgenerated points).

As a test-bed application, we are using a two-task experimental system developed at the US Naval Research Laboratory (NRL) in Washington D.C that has already been used for interruption-related studies. The primary (interrupted) task is a resource-allocation task named Three-Strike (TS). The objective is to attack and destroy three destinations utilizing available resources, ten heavy and ten light tanks and a certain amount of fuel and munitions. On their missions, users are encountering resistance from differing locales and different kinds of obstacles based on a stochastic model of the TS task. User's interactions are limited to mouse point-and-click events with several dialog-box style windows.

The interrupting task is Tactical Assessment (TA) task presented to a user at random points while she is performing the primary task. In this task, the user plays the role of a fighter aircraft pilot looking at a radar-screen-like display where three types of objects appear. The objective is to indicate whether the approaching object is hostile or neutral. The decision of the "pilot" is assisted by an intelligent-automated component that colors the objects as red (hostile), blue (neutral) or yellow (when the assessment can not be made). The user is to confirm the red/blue indications or give the appropriate classification for the yellow objects based on a set of rules. User's interactions consist of two keystrokes per object using the right-hand numeric key pad. The first keystroke is the object classification (5-neutral, 6-hostile) and the second is the track number that appears next to each object. 


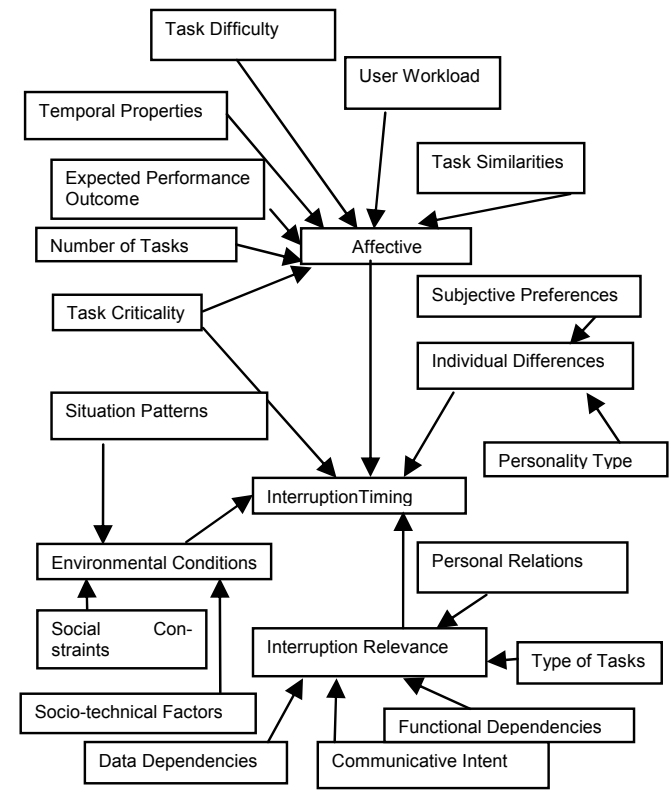

Fig. 2. High-level dependencies between factors for inferring the timing of interruption.

The complex structure of the primary (TS) task was divided into a set of subtasks with different sensitivity to interruption (e.g., outfitting tanks, allocating tanks to mission, planning a mission, carry out a mission and review the mission report). We have conducted a pilot study to help us identify the subtasks particularly sensitive to interruption and distinguish the interruption points that were denoted as obtrusive or during the pilot study and domain-specific knowledge were used as a basis for coupling and chunking the TS task structure. The results had implications for selection of taxonomic factors relevant in our experimental environment and for initialization and off-line training of the constructed BBN.

We would like to note that our exploratory study was also used as a first step in refining the interruption model and putting the emphasis on the task-related taxonomy categories. For this particular experiment the timing of the interruption was mapped and coded to a certain interruption point within subtask (e.g., before or after a particular interaction event). The system keeps records of the times and contexts of all relevant interaction events. Each time the specified situation arises (i.e., sequence of interaction events), the system infers the user's goals based on the context of her interaction before and at the time of the interruption. The system adjusts in such a way that a user is not interrupted during interruption points sensitive to interruptions, deferring the interruption task for the next opportune moment. 


\subsection{Pilot Study}

Fifteen volunteers participated in the pilot study using the same scenarios as experimental trials. Primary task resumption time defined as time from completing the interrupting task (restoring the TS window) to resuming the primary task was chosen as an appropriate measure in this study. Subjective preferences and perceptions were collected, but they were not considered as a factor in our implementation of the interruption coordination. Subjective preferences were generally inclined toward choosing the end of a subtask as the most opportune interruption point within each subtask. Not surprisingly, subjective preferences were not supported by actual objective measures. To the contrary, an interruption point placed at the end of a subtask led to longer resumption times partially because of the effort to decide on what to do next. Subjective preferences should not be neglected when designing user interfaces that give equal priority to user's satisfaction and comfort as to other performance measures.

Pilot study users were asked to rate the relative difficulty level of each subtask. The cognitive complexity/demand scale ranged from 1 (neglective) to 5 (very high). "Planning a mission" was rated as the most complex 4.5 , while "allocating a task to a mission" was rated as a subtask with almost neglective cognitive demand 1.3. Objective measures have confirmed that "planning a mission" subtask was most sensitive to interruptions. This subtask consists of three activities, reviewing the map, reviewing the destination status and selecting a destination. The results showed that interrupting a user during this subtask leads to prolonged resumption time, several times longer than the user's average inter-click time. The severe degree of disruption during the "planning" block was expected because of its complexity and user's cognitive load associated with it. What was perhaps more informative for our design was that interruptions at the beginning of subtasks subsequent to the "planning" block caused lengthy resumption times as well. The likeliest explanation could be that the user was still maintaining the "planning" goal state as a basis for subsequent actions (e.g., outfitting a tank depended on users' strategy and plans established during the "planning" stage).

This finding is in line with, but also somewhat contradictory to the results and suggestions presented in [1], [3] and [7]. Their results showed that interruption coming at the beginning of a task is less disruptive than those presented at later points. The contradictory results may be due to differences in our experimental designs, especially the types of tasks that were used. Their suggestions that the most opportune moment for interruptions could be the moments associated with a start of a task could be generalized to tasks similar to their experimental tasks. In general, relatively simple tasks without any interleaving and inter-relationships were used, namely simple VCR programming tasks in [7], sequence of independent web-search tasks in [1], and [3]. Real-life task are not necessarily independent of each other and domain specific knowledge impacts interaction in profound way that have to be incorporated in the mechanisms for coordinating interruptions. 


\subsection{Experiment in Progress}

A more formal experiment is currently underway. Twenty four participants were run through the experiment and the results presented in this section represent the initial data results. Each participant was instructed and trained on the primary and the interruption task, independently, followed by a $20 \mathrm{~min}$. practice trial in the context of interruptions. The practice session lasted for approximately 1 hour including the time for completing the background questionnaire. After short break, the participants concluded four 15-minute trials of the TS task interrupted by a number of times, either 8 or 12 , by the interruption task. The data were analyzed with a 2 (immediate and mediated interruption control) x 2 (8 and 12 number of interruptions during $15 \mathrm{~min}$. session) within-subject Analysis of Variance (ANOVA). We analyzed the withinsubject difference in task performance between the condition with immediate and computer-mediated coordination of interruption. An effort was made to control the number and the type of situations in which an interruption occurs keeping the frequency of interruptions randomly distributed across condition. Dependent variables were primary task resumption time, and accuracy on the interrupting task (i.e., percentage of correct object classifications).

Initial results have showed that resumption time for the interrupted task is sensitive to mediated coordination of interruption. Resumption times averaged at $5.5 \mathrm{sec}$ for immediate condition to $4.7 \mathrm{sec}$ for mediated coordination of interruptions. Fig. 3 contains mean resumption times separated by condition.

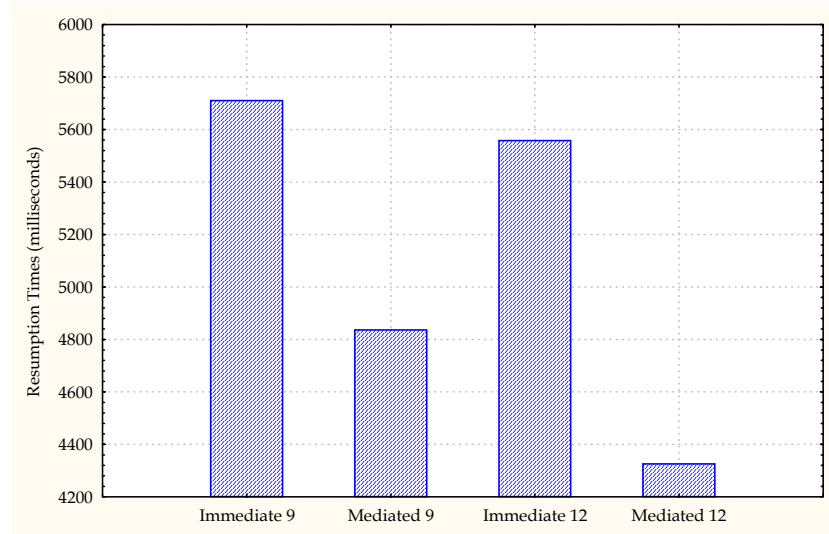

Fig. 3. Means of resumption times separated by condition

Significant main effects were observed both for coordination method of interruption, $\mathrm{F}(1,23)=48.707, \mathrm{p}<0.00001$, and frequency of interruptions $\mathrm{F}(1,23)=9.85$, $\mathrm{p}=0.0046$. No reliable interaction between coordination method and frequency of interruption was found. The results confirm the hypothesized performance advantage of taxonomy-based mediation of interruption resulting in resumption times compared 
to condition with immediate coordination method. We found no significance effect of coordination method of interruption and the frequency of interruption on user's accuracy when performing the interrupting task.

Subjective exit questionnaires were distributed at the end of the experimental trials to measure the subjective level of stress and anxiety experienced by a user at specific interruption points. Subsequent analysis will assess the effects of individual differences and any potential correlation between subjective and objective measures.

\section{Conclusions}

In this paper, we presented a taxonomy-based conceptual framework for a computersupported coordination of human interruptions in HCI. The outlined Interruption Taxonomy, which synthesizes various kinds of interruption-related context information served as a basis for implementing graceful coordination of interruption. An exploratory user study is underway to show the expressiveness and the effectiveness of the proposed framework. The initial results support the hypothesized performance advantage of taxonomy-based mediation of interruption to lessen the disruptive consequences of interruptions reflected in shorter resumption times compared to condition with immediate coordination method.

\section{References}

1. Bailey, B.P., Konstan J.A. and Carlis J.V.: The effects of interruptions on task performance, annoyance, and anxiety in the user interface. In: Proc. of Human-Computer Interaction INTERACT 2001. IOS Press, (2001) 593-601

2. Cellier, J-M., and Eyrolle, H.: Interference between switched tasks. Ergonomics, Vol. 35, No. 1, (1992) 25-36

3. Cutrell E. B., Czerwinski M. and Horvitz E.: Effects of instant messaging interruptions on computing tasks. In: Proc. of Conference on Human factors in computing systems - CHI 2000, ACM Press New York (2000) 99-100

4. Horvitz, E., Jacobs, A., and Hove, D.: Attention-sensitive alerting. In: Proc. of UAI '99, ACM Press (1999) 305-313

5. Kirmeyer, S.: Coping with competing demands: Interruption and the type a pattern. Journal of Applied Psychology, Vol. 73, No. 4 (1988) 621-629

6. Malone, T., and Crowston, K.: The interdisciplinary study of coordination. ACM Computing Surveys, Vol. 26, No. 1 (1994) 87-119

7. Monk C. A., Boehm-Davis D. A. and Trafton J. G.: The attentional costs of interrupting task performance at various stages. In: Proc. of 46th Annual Meeting of the Human Factors and Ergonomics Society, HFES (2002) 1824-1828 